\title{
Band structure of topological insulators from noise measurements in tunnel junctions
}

\author{
Juan Pedro Cascales, ${ }^{1, a)}$ Isidoro Martínez, ${ }^{1}$ Ferhat Katmis, ${ }^{2,3}$ Cui-Zu Chang, ${ }^{2}$ \\ Rubén Guerrero, ${ }^{4}$ Jagadeesh S. Moodera, ${ }^{2,3}$ and Farkhad G. Aliev ${ }^{1, b}$ \\ ${ }^{1}$ Dpto. Fisica Materia Condensada C3, Instituto Nicolas Cabrera (INC), Condensed Matter Physics Institute \\ (IFIMAC), Universidad Autonoma de Madrid, Madrid 28049, Spain \\ ${ }^{2}$ Department of Physics, Massachusetts Institute of Technology, Cambridge, Massachusetts 02139, USA \\ ${ }^{3}$ Francis Bitter Magnet Laboratory, Massachusetts Institute of Technology, Cambridge, Massachusetts 02139, \\ USA \\ ${ }^{4}$ Instituto Madrileño de Estudios Avanzados en Nanociencia (IMDEA-Nanociencia), Cantoblanco, \\ 28049 Madrid, Spain
}

(Received 4 September 2015; accepted 6 December 2015; published online 22 December 2015)

The unique properties of spin-polarized surface or edge states in topological insulators (TIs) make these quantum coherent systems interesting from the point of view of both fundamental physics and their implementation in low power spintronic devices. Here we present such a study in TIs, through tunneling and noise spectroscopy utilizing $\mathrm{TI} / \mathrm{Al}_{2} \mathrm{O}_{3} / \mathrm{Co}$ tunnel junctions with bottom TI electrodes of either $\mathrm{Bi}_{2} \mathrm{Te}_{3}$ or $\mathrm{Bi}_{2} \mathrm{Se}_{3}$. We demonstrate that features related to the band structure of the TI materials show up in the tunneling conductance and even more clearly through low frequency noise measurements. The bias dependence of $1 / \mathrm{f}$ noise reveals peaks at specific energies corresponding to band structure features of the TI. TI tunnel junctions could thus simplify the study of the properties of such quantum coherent systems that can further lead to the manipulation of their spin-polarized properties for technological purposes. (C) 2015 AIP Publishing LLC.

[http://dx.doi.org/10.1063/1.4938243]

A topological insulator (TI) is a material which is insulating in the bulk but presents spin-dependent conducting edge or surface states which are protected by time-reversal symmetry. ${ }^{1-3}$ A 2 D or 3D TI presents edge or surface states, respectively, which are spin-polarized in-plane, and locked at right angles to the carrier momentum, so that electrons with spin-up/down propagate in opposite directions. The edge or surface states of a TI consist of an odd number of massless Dirac cones. These properties along with their high mobility make TI materials interesting for next generation, low dissipation, and spintronic applications ${ }^{4,5}$ in which the electron spins are manipulated even without any magnetic fields. The experimental surge regarding these materials occurred with the prediction of Bi-based $\mathrm{TIs}^{6}$ and their posterior experimental realization. ${ }^{7} \mathrm{Bi}_{2} \mathrm{Se}_{3}$ and $\mathrm{Bi}_{2} \mathrm{Te}_{3}$, in particular, became the prototypical TI materials that were studied most heavily.

To date, the experimental verification of the band structure of TI materials has been predominantly carried out by angle-resolved photoemission spectroscopy (ARPES), which yields energy-momentum graphs of band dispersion for probing depths of under a few nm. ${ }^{7,8}$ Also, the use of spin-ARPES has allowed the determination of the spin dependence of the topological surface states. ${ }^{9}$ On the other hand, the use of scanning tunneling microscope (STM) allows obtaining information regarding the local density of states (DOS) and the topography of surfaces. By the study of quasiparticle scattering with STM, bands can be mapped very close to the Fermi surface, with a considerably lower energy range and at a

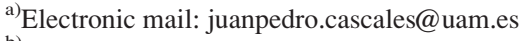

b)Electronic mail: farkhad.aliev@uam.es
}

smaller scale than with ARPES. ${ }^{10-13}$ Although immensely useful, these techniques are usually cumbersome and the conditions of study are far from a practical application of TIs. A versatile and relatively simple technique to determine the DOS of TI materials could be the study of electronic transport and noise through planar tunneling devices. So far, individual or heterostructure devices with TI layers have mainly dealt with lateral electron transport. ${ }^{14-23}$ For certain spintronic applications, perpendicular transport may be required for which a direct or indirect contact between the TI and other ferromagnetic layers is needed. Determining the band structure of TI films buried in multilayer structures as well as confirming the robustness of their electronic properties remains an unresolved, central issue for the possible technological application of TIs in spintronic devices. Characterizing the bias dependence of low frequency noise in tunnel junctions can be a useful tool to gather information on the band structure of buried interfaces, ${ }^{24}$ proving to be more sensitive to the opening and closing of transmission channels than transport or inelastic tunneling spectroscopy (IETS) measurements.

In this letter we report on the investigation of the band structure of the TI electrodes in perpendicular tunnel junctions by both electron transport and low frequency noise spectroscopy. Having a perpendicular tunneling transport allows probing the DOS of both the surface states and the bulk bands of the materials. The bottom TI electrodes of the samples are $\mathrm{Bi}_{2} \mathrm{Te}_{3}$ and $\mathrm{Bi}_{2} \mathrm{Se}_{3}$ with thicknesses of 10 and 20 quintuple layers (QL), well into the 3D TI range. ${ }^{25,26} \mathrm{~A}$ schematic diagram of the sample structure is shown in Fig. 1(a). In $\mathrm{Bi}_{2} \mathrm{Te}_{3}$, the Fermi energy lies within the conduction band and the Dirac point of the surface states is in the valence band. $\mathrm{Bi}_{2} \mathrm{Te}_{3}$ presents a similar band structure, but the Dirac 

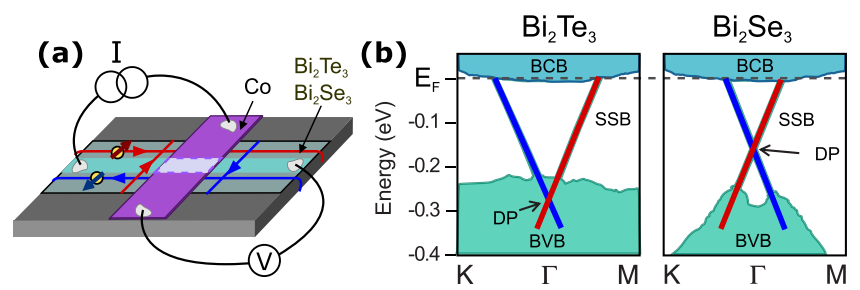

FIG. 1. (a) Diagram of the sample structure. (b) Diagram of the band structure of $\mathrm{Bi}_{2} \mathrm{Te}_{3}$ and $\mathrm{Bi}_{2} \mathrm{Se}_{3}$, adapted from Refs. 27 and 28 .

point is located between the valence and conduction bands. A schematic diagram of the band structure, adapted from ARPES results of $\mathrm{Bi}_{2} \mathrm{Te}_{3}{ }^{27}$ and $\mathrm{Bi}_{2} \mathrm{Se}_{3},{ }^{28}$ is shown in Fig. 1(b), where BCB stands for bulk conductance band, BVB for bulk valence band, SSB for surface state band, and DP for Dirac point.

The $\mathrm{Bi}_{2} \mathrm{Te}_{3}$ samples on $\mathrm{Si}(111)$ substrates have a layer structure of: $\mathrm{Bi}_{2} \mathrm{Te}_{3}(10 \quad \mathrm{QL}) / \mathrm{Al}_{2} \mathrm{O}_{3}(5.8 \mathrm{~nm}) / \mathrm{Co}(100 \mathrm{~nm}) /$ $\mathrm{AlO}_{x}(3.8 \mathrm{~nm})$. In order to improve the TI/barrier interface, we then employed (0001)-oriented, epi-ready, commercial $\mathrm{Al}_{2} \mathrm{O}_{3}$ (sapphire) substrates over which a thicker, $20 \mathrm{QL}$ film of $\mathrm{Bi}_{2} \mathrm{Se}_{3}$ was grown. The layer sequence for the $\mathrm{Bi}_{2} \mathrm{Se}_{3}$ junctions on sapphire substrates is $\mathrm{Bi}_{2} \mathrm{Se}_{3}(20 \mathrm{QL}) / \mathrm{Al}_{2} \mathrm{O}_{3}(5.7 \mathrm{~nm}) /$ $\mathrm{Co}(100 \mathrm{~nm}) / \mathrm{AlO}_{x}(3.8 \mathrm{~nm})$. The TI thin film growth was carried out by a molecular beam epitaxy (MBE) apparatus under ultra-high vacuum environment $\left(10^{-9}-10^{-10}\right.$ Torr). High purity $(5 \mathrm{~N})$ elemental $\mathrm{Bi}, \mathrm{Te}$, and $\mathrm{Se}$ were evaporated at growth rates between 0.5 and $1 \mathrm{~nm} / \mathrm{min}$, after which the film was annealed for an hour at $600^{\circ} \mathrm{C}$, and at $800^{\circ} \mathrm{C}$ for $30 \mathrm{~min}$ under $10^{-9}$ Torr. In-situ reflection high-energy electron diffraction (RHEED) monitorization (Fig. 2(b) inset) indicates a good two-dimensional growth. The X-ray diffraction (XRD) pattern $(\omega-2 \theta)$ of the films along the growth direction for $20 \mathrm{QL} \mathrm{Bi}_{2} \mathrm{Se}_{3}$ (in red) and $\mathrm{Bi}_{2} \mathrm{Te}_{3}$ (in black) is shown in Fig. 2(a). Laue oscillations around the Bragg peaks ((0003) and (0006)) are a clear indication of the film's structural coherence along the growth direction all the way to the top surface. Fig. 2(b) shows a rocking curve ( $\omega$-scan) on each Bragg peak and one of the (0006)-peaks, where Gaussian broadening is caused by finite size effects and an additional Lorentzian (a)

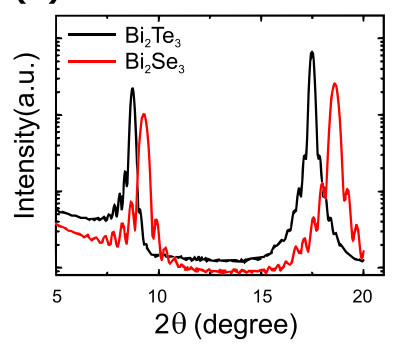

(b)

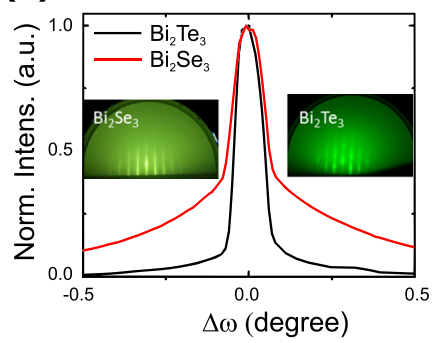

FIG. 2. $\mathrm{Cu}-\mathrm{K}_{\alpha}$ radiation and $15 \mathrm{keV}$ electron beam are used for XRD and RHEED measurements, respectively. (a) Typical XRD pattern for symmetric Bragg reflections of $\mathrm{Bi}_{2} \mathrm{Se}_{3}$ (in red) and $\mathrm{Bi}_{2} \mathrm{Te}_{3}$ (in black). (0001)-type reflections are visible with pronounced thickness fringes at the vicinity of the layer reflections which shows a (0001)-type layer orientation. The fringes along the out-of-plane direction are due to the layer thickness and the quality of the film. (b) In-plane line-cut for symmetric (0006) reflection for both types of TI films. The rocking curve for (0006) Bragg peak is taken along the $\langle\overline{1010}\rangle$ crystallographic direction of the substrate. The insets show the RHEED images for both TI films. lineshape appears due to defects, such as vacancies, dislocations, etc. By fitting a pseudo-Voigt function to the curves, we can conclude that the defect density within $\mathrm{Bi}_{2} \mathrm{Se}_{3}$ is larger than for $\mathrm{Bi}_{2} \mathrm{Te}_{3}$.

The transport and low frequency noise setup was described in the previous articles. ${ }^{29,30}$ Electrons tunnel from the Co (TI) to the TI (Co) for positive (negative) bias. Fig. 3 presents low temperature, transport measurements carried out on a $1.8 \mathrm{k} \Omega \mathrm{Bi}_{2} \mathrm{Te}_{3}$ sample. A fit of the Brinkman's ${ }^{31}$ (not shown) to the sample's IV at $\mathrm{T}=0.3 \mathrm{~K}$ with yields an effective tunnel barrier thickness of around $1.5 \mathrm{~nm}$. This could imply a rough $\mathrm{TI} /$ barrier interface, since the nominal barrier is $5.8 \mathrm{~nm}$ thick. As can be seen in Fig. 3(a), the conductance presents a parabolic-like dependence (junction-like behavior) with changes in slope at energies which could be related to features in the DOS of the TI. The overall shape of the conductance did not change with the temperature, except for a zero-bias anomaly (ZBA) peak which accentuated with decreasing temperature. This could mean that the surface states are robust at least up to $90 \mathrm{~K}$. The slope changes in the conductance could correspond, as was discussed in Ref. 10, to leaving and entering the different bands of the materials: conductance band, surface state, and valence band.

$1 / \mathrm{f}$ noise is described by the power spectral density $S_{V}(f)=\frac{\alpha V^{2}}{A f^{\beta}}$ (Ref. 32) where $V$ is the applied bias, $A$ the junction area, and $\beta \sim 1$. The normalized $1 /$ f noise or Hooge parameter $\alpha$ is extracted from the spectra by performing the linear fit $\log \left(S_{V}(f)\right)=\log \left(\frac{\alpha V^{2}}{A}\right)-\beta \log (f)$. As is shown in Fig. 3(b), $\alpha$ presents several clear peaks at certain values of energy which compare favorably with inflection points in the conductance curves, shown as arrows in Fig. 3(a) and as triangles in Fig. 3(b). The energies are estimated from the IETS spectrum in Fig. 4(a), presenting features which could arise from phonons in the $\mathrm{AlO}_{x},{ }^{33}$ the $\mathrm{TI},{ }^{34}$ or coupled $\mathrm{AlO}_{x^{-}}$ TI modes. The occurrence of inelastic tunneling processes with the addition of sequential tunneling observed in shot
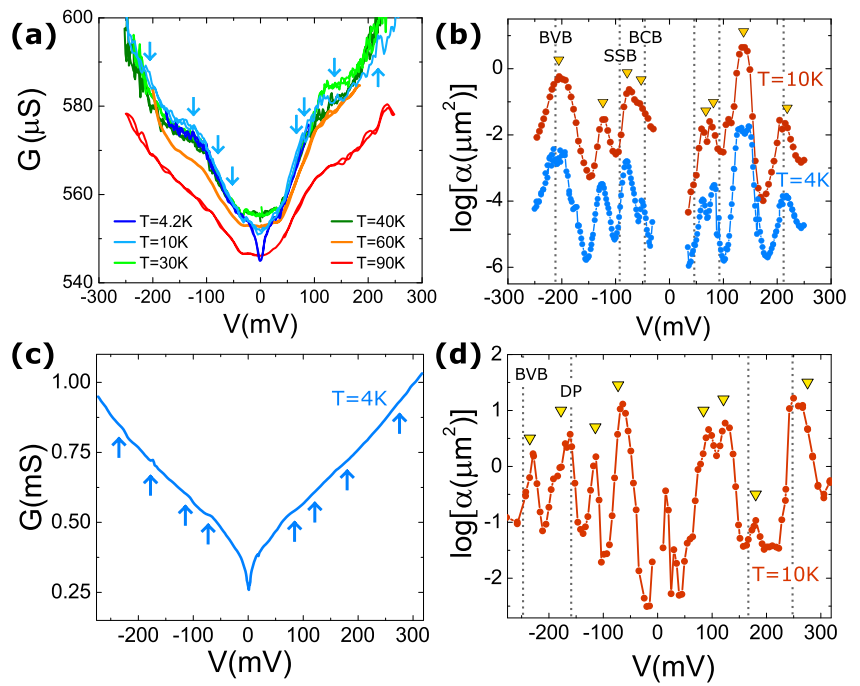

FIG. 3. (a) Conductance measurements at different temperatures for a $\mathrm{Bi}_{2} \mathrm{Te}_{3} / \mathrm{AlO}_{x} / \mathrm{Co}$ tunnel junction. (b) $1 /$ f noise $v s$. bias in the $\mathrm{Bi}_{2} \mathrm{Te}_{3}$ junction for two temperatures (curves offset for convenience), correlated with inflection points of the conductance (triangles) and $\mathrm{Bi}_{2} \mathrm{Te}_{3}$ band features (dashed lines). ${ }^{10}$ (c) Conductance at $\mathrm{T}=4 \mathrm{~K}$ for $\mathrm{a}_{\mathrm{Bi}} \mathrm{Se}_{3} / \mathrm{AlO}_{x} / \mathrm{Co}$ tunnel junction and (d) $1 / \mathrm{f}$ noise at $\mathrm{T}=10 \mathrm{~K}$, compared to conductance inflection points (triangles) and $\mathrm{Bi}_{2} \mathrm{Se}_{3}$ band features (dashed lines). ${ }^{28}$ 

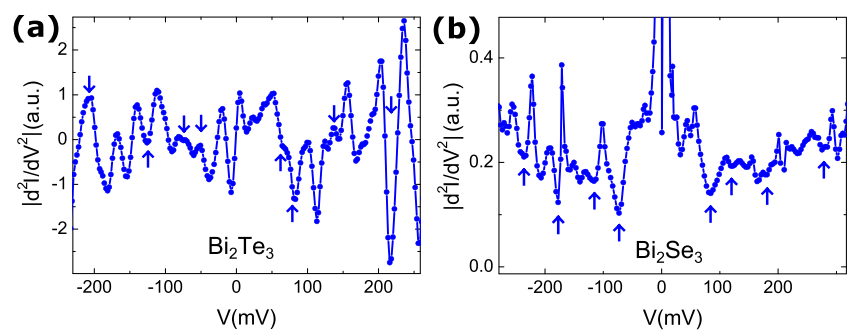

FIG. 4. Derivative (in absolute value) of the conductance at $\mathrm{T}=4 \mathrm{~K}$ of (a) $\mathrm{Bi}_{2} \mathrm{Te}_{3}$ and (b) $\mathrm{Bi}_{2} \mathrm{Se}_{3}$ junctions. Arrows indicate the bias of maxima or minima close to peaks in $1 / \mathrm{f}$.

noise measurements (not shown) could lead to a fraction of the carriers being inelastically back-scattered against the flow of the current. ${ }^{35}$ This could explain why the peaks in $1 / f$ noise are observed for both signs of the bias. Furthermore, these energies can be seen to relate to the band structure of the TI obtained by ARPES and STM in Ref. 10, shown by dashed lines. The $1 / \mathrm{f}$ peak around $\pm 200 \mathrm{mV}$ is close to the value for the beginning of the valence band ${ }^{10}$ (BVB). The remaining peaks could be due to the opening TI SSB and the $\mathrm{BCB}$. These features may originate from the opening or closing of transport channels which influences the voltage fluctuations, as shown in Ref. 24. The temperature dependence of the peaks around $-100 \mathrm{mV}$ was tracked (not shown) when the sample was allowed to warm up from $\mathrm{LHe}^{4}$ temperatures. The band-related features gradually disappear when the temperature is increased. However, the position of both peaks, specially the one around $-120 \mathrm{mV}$, does not shift in energy when the temperature increases. This may provide an argument that these features are related to the surface states, which have been reported to be thermally stable. ${ }^{36}$

Similar results were obtained for the junctions with a 20 QL $\mathrm{Bi}_{2} \mathrm{Se}_{3}$ electrode. Figs. 3(c) and 3(d) present the results obtained for a $4.2 \mathrm{k} \Omega$ junction. The dependence of the conductance with the bias is shown in Fig. 3(c), which is parabolic-like and presents changes in slope, which can be estimated by differentiating the conductance curve (Fig. 4(b)). We indicate the most relevant inflection points for $1 / \mathrm{f}$ results by arrows. The analysis of $1 / \mathrm{f}$ noise also presents peaks which appear at different energies (see Fig. 3(d)). Inflection points in the conductance from Fig. 4(b), which are close in energy to the peaks in $1 / \mathrm{f}$, are indicated by triangles and compared to the band features of the TI from Ref. 7. The peak located around \pm 250 could be related to the beginning of the valence band (BVB). The feature located around $\pm 160 \mathrm{mV}$ could be related to the DP. The remaining peaks in $1 / \mathrm{f}$ noise could be due to the opening of the surface state band and the conductance band, as in the $\mathrm{Bi}_{2} \mathrm{Te}_{3}$ sample.

Even though evidence of transport through the TI surface states is found, we did not observe a magnetoresistive response with fields up to $3 \mathrm{kOe}$. Since the bulk DOS of TIs is not polarized in spin, the Zeeman splitting caused by the 3 kOe field might not be enough to observe any significant effect. Whereas the absence of the surface driven spin polarization in the TI response may indicate a loss of spin polarization through spin scattering at the interfaces or defects, ${ }^{37-39}$ the ZBA seen in the conductance $v s$ bias may relate to loss of spin information due to $\mathrm{Co}$ atoms inside the $\mathrm{Al}_{2} \mathrm{O}_{3}$ barrier which acts as scattering centers or to higher surface defect densities of the TI, as shown by XRD.

In conclusion, we report the fabrication of tunnel junctions with a TI bottom electrode. We have demonstrated that features related to the band structure of these materials in contact with ferromagnetic layers through an $\mathrm{Al}_{2} \mathrm{O}_{3}$ barrier can be experimentally detected through electronic transport measurements and even more clearly through low frequency noise measurements. We do not exclude the influence of inelastic scattering by barrier and interface related phonons. ${ }^{40}$ One way to confirm our results could be to look for peaks in $1 / \mathrm{f}$ noise in $\mathrm{Al} / \mathrm{Al}_{2} \mathrm{O}_{3} / \mathrm{Bi}$ junctions as $\mathrm{Bi}$ shows band features in the conductance $v s$ bias. Obtaining reliable, TI tunnel junctions could simplify the study of their properties and pave the way for the manipulation of their spinpolarized properties for technological purposes. Future measurements will deal with TI films in the 2D limit, with the aim of exploiting the spin polarized edge states for spintronic applications. An interesting alternative would be to carry out noise measurements in $p n$ junctions with a TI semiconductor. ${ }^{41}$ Also, crystalline barriers such as $\mathrm{MgO}$ could improve the interface and allow the probing of the surface state spin texture.

F.A. and I.M. acknowledge support by the Spanish MINECO (MAT2012-32743) and the Comunidad de Madrid through NANOFRONTMAG-CM (S2013/MIT-2850). J.P.C. acknowledges support from the Fundacion Seneca (Region de Murcia) posdoctoral fellowship (19791/PD/15) and J.S.M., C.Z.C., and F.K. from Grants NSF (DMR-1207469), ONR (N00014-13-1-0301), and the STC Center for Integrated Quantum Materials under NSF Grant DMR-1231319.

${ }^{1}$ C. L. Kane and E. J. Mele, Phys. Rev. Lett. 95, 146802 (2005).

${ }^{2}$ L. Fu, C. L. Kane, and E. J. Mele, Phys. Rev. Lett. 98, 106803 (2007).

${ }^{3}$ M. König, S. Wiedmann, C. Brüne, A. Roth, H. Buhmann, L. W. Molenkamp, X.-L. Qi, and S.-C. Zhang, Science 318, 766 (2007).

${ }^{4}$ C.-Z. Chang, W. Zhao, D. Y. Kim, H. Zhang, B. A. Assaf, D. Heiman, S.C. Zhang, C. Liu, M. H. Chan, and J. S. Moodera, Nat. Mater. 13, 473-477 (2015)

${ }^{5}$ M. Götte, T. Paananen, G. Reiss, and T. Dahm, Phys. Rev. Appl. 2, 054010 (2014).

${ }^{6}$ H. Zhang, C.-X. Liu, X.-L. Qi, X. Dai, Z. Fang, and S.-C. Zhang, Nat. Phys. 5, 438 (2009).

${ }^{7}$ Y. Xia, D. Qian, D. Hsieh, L. Wray, A. Pal, H. Lin, A. Bansil, D. Grauer, Y. Hor, R. Cava et al., Nat. Phys. 5, 398 (2009).

${ }^{8}$ D. Hsieh, D. Qian, L. Wray, Y. Xia, Y. S. Hor, R. Cava, and M. Z. Hasan, Nature 452, 970 (2008).

${ }^{9}$ C. Jozwiak, C.-H. Park, K. Gotlieb, C. Hwang, D.-H. Lee, S. G. Louie, J. D. Denlinger, C. R. Rotundu, R. J. Birgeneau, Z. Hussain et al., Nat. Phys. 9, 293 (2013).

${ }^{10}$ Z. Alpichshev, J. G. Analytis, J.-H. Chu, I. R. Fisher, Y. L. Chen, Z. X. Shen, A. Fang, and A. Kapitulnik, Phys. Rev. Lett. 104, 016401 (2010).

${ }^{11}$ T. Zhang, P. Cheng, X. Chen, J.-F. Jia, X. Ma, K. He, L. Wang, H. Zhang, X. Dai, Z. Fang et al.. Phys. Rev. Lett. 103, 266803 (2009).

${ }^{12}$ P. Cheng, C. Song, T. Zhang, Y. Zhang, Y. Wang, J.-F. Jia, J. Wang, Y. Wang, B.-F. Zhu, X. Chen et al., Phys. Rev. Lett. 105, 076801 (2010).

${ }^{13}$ T. Hanaguri, K. Igarashi, M. Kawamura, H. Takagi, and T. Sasagawa, Phys. Rev. B 82, 081305 (2010).

${ }^{14}$ J. G. Checkelsky, Y. S. Hor, R. J. Cava, and N. P. Ong, Phys. Rev. Lett. 106, 196801 (2011).

${ }^{15}$ D. Kong, Y. Chen, J. J. Cha, Q. Zhang, J. G. Analytis, K. Lai, Z. Liu, S. S. Hong, K. J. Koski, S.-K. Mo et al., Nat. Nanotechnol. 6, 705 (2011).

${ }^{16}$ S. Cho, N. P. Butch, J. Paglione, and M. S. Fuhrer, Nano Lett. 11, 1925 (2011).

${ }^{17}$ C. Li, O. vant Erve, J. Robinson, Y. Liu, L. Li, and B. Jonker, Nat. Nanotechnol. 9, 218 (2014). 
${ }^{18}$ B. A. Assaf, F. Katmis, P. Wei, B. Satpati, Z. Zhang, S. P. Bennett, V. G. Harris, J. S. Moodera, and D. Heiman, Appl. Phys. Lett. 105, 102108 (2014).

${ }^{19}$ B. A. Assaf, F. Katmis, P. Wei, C.-Z. Chang, B. Satpati, J. S. Moodera, and D. Heiman, Phys. Rev. B 91, 195310 (2015).

${ }^{20}$ A. D. Liao, M. Yao, F. Katmis, M. Li, S. Tang, J. S. Moodera, C. Opeil, and M. S. Dresselhaus, Appl. Phys. Lett. 105, 063114 (2014).

${ }^{21}$ Z. Jiang, F. Katmis, C. Tang, P. Wei, J. S. Moodera, and J. Shi, Appl. Phys. Lett. 104, 222409 (2014).

${ }^{22}$ P. Wei, F. Katmis, B. A. Assaf, H. Steinberg, P. Jarillo-Herrero, D. Heiman, and J. S. Moodera, Phys. Rev. Lett. 110, 186807 (2013).

${ }^{23}$ B. A. Assaf, T. Cardinal, P. Wei, F. Katmis, J. S. Moodera, and D. Heiman, Appl. Phys. Lett. 102, 012102 (2013).

${ }^{24}$ F. G. Aliev, J. P. Cascales, A. Hallal, M. Chshiev, and S. Andrieu, Phys Rev. Lett. 112, 216801 (2014).

${ }^{25}$ C.-X. Liu, H. Zhang, B. Yan, X.-L. Qi, T. Frauenheim, X. Dai, Z. Fang, and S.-C. Zhang, Phys. Rev. B 81, 041307 (2010).

${ }^{26}$ Y. Zhang, K. He, C.-Z. Chang, C.-L. Song, L.-L. Wang, X. Chen, J.-F. Jia, Z. Fang, X. Dai, W.-Y. Shan et al., Nat. Phys. 6, 584 (2010).

${ }^{27}$ J. Zhang, C.-Z. Chang, Z. Zhang, J. Wen, X. Feng, K. Li, M. Liu, K. He, L. Wang, X. Chen et al., Nat. Commun. 2, 574 (2011).

${ }^{28}$ Y. Zhao, C.-Z. Chang, Y. Jiang, A. DaSilva, Y. Sun, H. Wang, Y. Xing, Y. Wang, K. He, X. Ma et al., Sci. Rep. 3, 3060 (2013).
${ }^{29}$ R. Guerrero, F. G. Aliev, Y. Tserkovnyak, T. S. Santos, and J. S. Moodera, Phys. Rev. Lett. 97, 266602 (2006).

${ }^{30}$ R. Guerrero, D. Herranz, F. G. Aliev, F. Greullet, C. Tiusan, M. Hehn, and F. Montaigne, Appl. Phys. Lett. 91, 132504 (2007).

${ }^{31}$ W. Brinkman, R. Dynes, and J. Rowell, J. Appl. Phys. 41, 1915 (1970).

${ }^{32}$ F. N. Hooge, T. G. M. Kleinpenning, and L. K. J. Vandamme, Rep. Prog. Phys. 44, 479 (1981).

${ }^{33}$ R. A. Dragoset, E. S. Phillips, and R. V. Coleman, Phys. Rev. B 26, 5333-5346 (1982).

${ }^{34}$ X. Zhu, L. Santos, R. Sankar, S. Chikara, C. Howard, F. C. Chou, C. Chamon, and M. El-Batanouny, Phys. Rev. Lett. 107, 186102 (2011).

${ }^{35}$ M. Büttiker, Phys. Rev. B 33, 3020 (1986).

${ }^{36}$ L. Zhao, H. Deng, I. Korzhovska, Z. Chen, M. Konczykowski, A. Hruban, V. Oganesyan, and L. Krusin-Elbaum, Nat. Mater. 13, 580 (2014).

${ }^{37}$ J. S. Moodera, J. Nassar, and G. Mathon, Annu. Rev. Mater. Sci. 29, 381 (1999).

${ }^{38}$ G.-X. Miao, M. Münzenberg, and J. S. Moodera, Rep. Prog. Phys. 74, 036501 (2011).

${ }^{39}$ J. S. Moodera and R. H. Meservey, in Magnetoelectronics, edited by M. Johnson (Academic Press, San Diego, 2004), pp. 151-378.

${ }^{40}$ V. Drewello, J. Schmalhorst, A. Thomas, and G. Reiss, Phys. Rev. B 77, 014440 (2008).

${ }^{41}$ R. Yoshimi, A. Tsukazaki, K. Kikutake, J. G. Checkelsky, K. S. Takahashi, M. Kawasaki, and Y. Tokura, Nat. Mater. 13, 253 (2014). 